\title{
Notas sobre la enseñanza de alfabetización a inmigrantes adultos
}

\author{
MARCIN SOSINSKI \\ Universidad de Granada \\ sosinski@ugr.es \\ MARTHA YounG-SCHOLTEN \\ Newcastle University \\ martha.young-scholten@newcastle.ac.uk \\ ROLA NAEB \\ Northumbria University \\ rola.naeb@northumbria.ac.uk
}

Resumen: En este trabajo, a partir del análisis del concepto de alfabetización, se presentan brevemente cinco herramientas y metodologías para la adquisición de la lectoescritura por parte de inmigrantes adultos. La alfabetización es un proceso paulatino y los alumnos adultos que se alfabetizan en una nueva lengua tienen perfiles diversos, por lo tanto, es necesario proporcionarles apoyo específico, adaptado a sus necesidades. El método de palabra generadora y el Instructor Digital de Alfabetización se centran en la decodificación inicial de palabras. En cambio, el enfoque de experiencia lingüística y el proyecto de creación de libros de lectura Simple Stories son apropiados para mejorar la comprensión lectora. Por último, la Plataforma de Lenguas de Herencia aboga por el uso de la lengua materna de los alumnos.

Palabras clave: alfabetización, inmigrantes adultos, conciencia fonológica, lectura extensiva, lengua materna.

Abstract: In this paper, based on the analysis of the concept of literacy, five tools and methodologies for the literacy acquisition by adult immigrants are briefly introduced. Literacy is a gradual process and adult learners who become literate in a new language have different profiles, so it is necessary to provide them with specific support, adapted to their needs. The Generative Word method and the Digital Literacy Instructor focus on the initial decoding of words. On the other hand, the Language Experience Approach and the Simply Stories project for book creation are appropriate for improving reading comprehension. Finally, the Heritage Language Hub promotes the use of the learner's mother tongue.

Key words: literacy, adult immigrants, phonological awareness, extensive reading, mother tongue.

\section{Introducción}

El objetivo de este trabajo, en la línea del taller presentado en ForoELE de Valencia de 2020, es hacer una revisión fundamentada de algunas metodologías y herramientas utilizadas para la alfabetización de inmigrantes adultos, concediéndole una atención especial a la lectura. 
Nuestro punto de partida es la situación en la que, entre los adultos que inmigran a las sociedades postindustrializadas, hay un grupo especial de aquellos que tienen un bajo nivel de instrucción formal en su lengua materna, es decir, que no han desarrollado alguna o ninguna de las habilidades de las que consta la lectoescritura. Es una gran diferencia, frente a otros adultos en nuestro ámbito cultural que, por lo general, cuando empiezan el aprendizaje de una nueva lengua, ya están alfabetizados.

A estas personas se les suele denominar popularmente analfabetos o no alfabetizados, si bien conviene hacer unas precisiones acerca de los términos empleados. Según las definiciones proporcionadas en el glosario de la Asociación Internacional de Alfabetización (https://www.literacyworldwide.org), alfabetización es

La habilidad de reconocer, entender, interpretar, crear, procesar y comunicar, utilizando materiales visuales, sonoros y digitales, en cualquier ámbito y contexto. A lo largo del tiempo, alfabetización se ha aplicado a una amplia gama de actividades y se usa como alfabetización informática, matemática o dietética; en tales casos, se refiere a los conocimientos básicos de un ámbito, más que, específicamente, a la lectura y la escritura.

Nosotros nos referimos aquí a esa habilidad original, limitada tanto a la lectura, entendida como «el proceso de extracción y construcción simultánea de significado a través de la interacción y la implicación con el lenguaje escrito. Utilizamos las palabras "extraer y construir" para enfatizar tanto la importancia como la insuficiencia del texto como determinante de la comprensión de lectura», como a la escritura, definida como «el proceso de registrar el lenguaje gráficamente, a mano o por otros medios, por ejemplo, mediante letras, logogramas y otros símbolos».

Las definiciones aportadas explicitan que la alfabetización es una habilidad compleja, que involucra varias subhabilidades, y que la lectura, que tradicionalmente se ha catalogado como una destreza receptiva (VV. AA. 2000), es un proceso interactivo y original para el cual se necesita mucho más que el conocimiento del alfabeto.

Por la misma razón conviene matizar la distinción binaria entre alfabetizado/no alfabetizado y hablar de varias situaciones que recogemos en la siguiente tabla (Tabla 1) (Burt et al. 2008).

\begin{tabular}{|l|l|}
\hline Tipo & Características \\
\hline No alfabetizado & $\begin{array}{l}\text { Los alumnos no han tenido acceso a instrucción formal pero } \\
\text { sí está disponible en su país de origen }\end{array}$ \\
\hline Prealfabetizado & $\begin{array}{l}\text { La primera lengua del alumno no cuenta con forma escrita o } \\
\text { esta está en proceso de desarrollarse (por ejemplo, lenguas } \\
\text { indígenas americanas, africanas, de Australia o lenguas del } \\
\text { Pacífico que no tienen forma escrita) }\end{array}$ \\
\hline Semialfabetizado & $\begin{array}{l}\text { Los alumnos tienen un mínimo de alfabetización en su } \\
\text { lengua materna }\end{array}$ \\
\hline $\begin{array}{l}\text { Alfabetizados en escritura } \\
\text { no alfabética }\end{array}$ & $\begin{array}{l}\text { Los alumnos están alfabetizados en la lengua escrita, pero } \\
\text { en una forma de escritura no alfabética (por ejemplo, el } \\
\text { chino mandarín) }\end{array}$ \\
\hline
\end{tabular}




\begin{tabular}{|l|l|}
\hline $\begin{array}{l}\text { Alfabetizado en escritura } \\
\text { alfabética (no alfabeto } \\
\text { romano) }\end{array}$ & $\begin{array}{l}\text { Los alumnos están alfabetizados en la lengua escrita en un } \\
\text { alfabeto no romano (por ejemplo, el árabe, el griego, el } \\
\text { coreano, el ruso ol thai). } \\
\text { La dirección de la escritura puede también variar. }\end{array}$ \\
\hline $\begin{array}{l}\text { Alfabetizado en escritura } \\
\text { alfabética } \\
\text { romano) }\end{array}$ & $\begin{array}{l}\text { Los alumnos están alfabetizados en la lengua escrita en el } \\
\text { alfabeto romano (por ejemplo, el francés, el alemán y el } \\
\text { español). Leen de izquierda a derecha y reconocen la forma } \\
\text { de las letras y sus tipos. }\end{array}$ \\
\hline
\end{tabular}

Tabla 1: Clasificación de alumnos, según el tipo de alfabetización.

No es el objetivo del artículo ahondar en estas distinciones, pero, por ejemplo, alumnos no alfabetizados y prealfabetizados pueden parecen iguales en cuanto a su situación de partida — no poseen ningún dominio de la lectoescritura-, no obstante, los primeros están familiarizados con el concepto de la escritura, saben cuál es su función social y tal vez estén más motivados porque comprenden la necesidad de saber leer y escribir, así como conocen las ventajas de estar alfabetizado. La vida de los segundos, en cambio, se ha desarrollado en ausencia casi total del código escrito, por lo que no siempre experimentan la necesidad de alfabetizarse.

En este trabajo nos referiremos, sin distinguir, a las personas de los tres primeros grupos y los denominaremos también alumnos LESLLA porque los tres autores pertenecemos a un foro internacional con ese nombre, Literacy Education and Second Language and Literacy for Adults ${ }^{1}$ (véase www.leslla.org).

La causa más frecuente de su poca o ninguna escolarización formal son las condiciones socioeconómicas y políticas que los condujeron a la emigración. No obstante, como todos los inmigrantes, se espera de ellos que se integren en la sociedad en la que se han reasentado y, para ello, es necesario que sepan leer y escribir, e, incluso, que sean capaces de realizar pruebas de nivel en su nuevo idioma. En ese sentido, cabe señalar que, según un informe del Consejo de Europa (Rocca et al. 2019), de los 41 países que respondieron a la encuesta de 2018, la mayoría exige algún tipo de prueba lingüística, vinculada a los niveles MCER: $24 \%$ antes de la entrada en el territorio nacional, $32 \%$ para optar a la residencia temporal, $51 \%$ para obtener la residencia permanente y $78 \%$ para obtener la nacionalidad.

Por otra parte, las ventajas de haberse escolarizado, aunque sean unos pocos años, están bien documentadas: los alumnos alfabetizados y semialfabetizados en la lengua materna y en cualquier sistema de escritura progresan más rápidamente en el aprendizaje de una nueva lengua, mientras que los no alfabetizados lo hacen más lentamente (Condelli et al. 2003; Filimban 2019; Kurvers et al. 2010; Kurvers y van der Zouw 1990; Strucker y Davidson 2003; Tarone y Bigelow 2005; Young-Scholten y Naeb 2010; YoungScholten y Strom 2006). Se podría pensar que la lentitud del progreso de las personas con bajo nivel de alfabetización en la lengua materna, es decir, sin posibilidad de transferir a la nueva lengua las destrezas adquiridas, podría deberse a la edad en que se

\footnotetext{
${ }^{1}$ La asociación LESLLA se creó en 2005 con el objetivo de impulsar la idea de que, a través de la colaboración entre investigadores y profesionales, era posible mejorar el apoyo educativo a inmigrantes adultos no alfabetizados. Nótese que, paralelamente, más o menos en la misma época, se publican el Manifiesto de Santander (2004) y las Propuestas de Alicante (2006), así como se crea el portal segundaslenguaseinmigracion.com
} 
enfrentan al reto de aprender a leer por primera vez en su vida. Sin embargo, las investigaciones demuestran que la edad no es una barrera infranqueable. Se sabe ya bastante sobre cómo aprenden los niños a leer (Chall 1999; Ehri 1994; Goswami y Bryant 1990; Juel 1991; Perfetti 2010). Si comparamos los conocimientos previos a la adquisición de lectura de los niños y los adultos no alfabetizados, así como las etapas del desarrollo de la lectura, las similitudes que encontramos nos llevan a la conclusión de que esos últimos tienen la capacidad cognitiva para aprender a leer a lo largo de toda la vida (Kurvers 2002; Morais et al. 1988; Young-Scholten y Naeb 2010; YoungScholten y Strom 2006). Por lo tanto, el verdadero obstáculo sería el reto de adquirir competencia lingüística en un nuevo idioma en la edad adulta y esa es, en nuestra opinión, la causa de la lentitud del progreso de los no alfabetizados (Kurvers y Stockmann 2009; Tammelin-Laine 2014). De hecho, desde hace mucho tiempo se ha considerado que un período crítico (Lenneberg 1967) es el responsable de lo que se observa habitualmente: casi nadie, pasada la pubertad, logra adquirir plenamente una nueva lengua. Sin embargo, las investigaciones realizadas en este caso también muestran que no existe un período crítico para el vocabulario y que, a pesar de la falta de éxito completo, los estudiantes mayores siguen una ruta predecible, similar a la de los niños, que es en gran medida independiente de la edad de la exposición inicial, el idioma nativo, el tipo de exposición o los antecedentes educativos (Hawkins 2001). Las investigaciones sobre aprendientes adultos a lo largo de las décadas, comenzando con los trabajadores migrantes en Alemania, como Becker et al. (1977) y Clahsen et al. (1983), continuados por estudios de inmigrantes, como los de Vainikka y YoungScholten (2011), y el estudio multinacional de la Fundación Europea de la Ciencia (véase Klein y Perdue 1997), demuestran que los inmigrantes pueden alcanzar altos niveles de rendimiento si, en palabras de Krashen, el input es comprensible y la exposición es suficiente (Krashen 1985). No obstante, en el caso de los inmigrantes, a pesar de estar en el contexto de inmersión, el input no siempre es suficiente (Villalba 2010) y, si además no saben leer y escribir, no pueden apoyarse en la lengua escrita para aumentar su contacto con la nueva lengua.

\section{Apoyo educativo para alumnos no alfabetizados}

Fue en las décadas de 1970 y 1980, cuando, a nivel estatal y municipal, se empezó a ofrecer clases de idiomas y de alfabetización para inmigrantes; y aunque ahora hay más apoyo de ese tipo, su disponibilidad varía de un país a otro, así como varían las condiciones en las que se imparten esas clases y su contenido.

Los pocos estudios que relacionan los resultados alcanzados con lo que sucede en el aula sugieren que, en el caso de clases de alfabetización, dan buenos resultados los cursos que llevan el mundo exterior al aula, aquellos en los que hay tareas para hacer en casa y los que se basan en la instrucción individualizada (Alamprese 2009; Condelli et al. 2003; Durğunoglu 2012; Johnson y Berry 2014; Kurvers 2002; Kurvers et al. 2010; Olson y Torrance 2009; Reder y Bynner 2009; Shameem et al. 2002; Strucker y Davidson 2003). Además, Kurvers y van der Zouw (1990) demostraron la efectividad de cursos basados en los métodos fonéticos. Aparte de considerar el contenido y la metodología de la instrucción que reciben los estudiantes, también es importante considerar el número de horas y, en este aspecto, los autores comprobaron la efectividad de las clases intensivas. 
Por otra parte, los profesionales que trabajan con alumnos LESLLA han adaptado durante mucho tiempo ideas que han tenido éxito con otros alumnos, niños y adultos alfabetizados. Hay millones de estudiantes de idiomas extranjeros educados en todo el mundo y una industria que los atiende. Los enfoques, métodos, técnicas y materiales utilizados con esos estudiantes pueden ser y han sido adaptados para inmigrantes adultos. No obstante, por varias razones, son menos efectivos con los adultos no alfabetizados. En primer lugar, suponen la transferencia de las aptitudes de lectura y escritura de la lengua materna del educando: la educación formal cursada por alumnos alfabetizados les dota una serie de habilidades adicionales que van desde los fundamentos de cómo sostener un bolígrafo y cómo utilizar un ratón de ordenador hasta la reflexión sobre el lenguaje como un objeto (la conciencia metalingüística). Además, en cuanto al tratamiento de las áreas temáticas, los materiales educativos más populares y mejor diseñados están adaptados a alumnos que se supone que son de clase media y no tienen la intención de reasentarse en el país cuya lengua aprenden, con lo cual pueden no ser motivadores ni interesantes para inmigrantes adultos con un bajo nivel de instrucción que agradecen el tratamiento mucho más detallado de temas como la salud, la vivienda o el trabajo.

Evidentemente, los adultos no alfabetizados se benefician mejor de los enfoques, métodos, técnicas y materiales que se han diseñado específicamente para ellos, pero sin cometer el error de considerar que es necesaria una metodología radicalmente diferente (Villalba 2019), distinta del paradigma comunicativo imperante en nuestra época. En los siguientes párrafos, describimos cinco de esos métodos y herramientas. Los dos primeros se centran en la descodificación inicial/reconocimiento de palabras y dos últimos en la comprensión lectora. El quinto proyecto explora la posibilidad de usar en el aula la lengua materna de los alumnos. Esa presentación dista mucho de ser exhaustiva e instamos al lector a que visite las páginas web de referencia: leslla.org, segundaslenguaseinmigración.com o, específicamente, la página web del proyecto europeo EU-SPEAK, donde puede leer en español sobre un proyecto de formación y desarrollo de profesores de inmigrantes adultos no alfabetizados: https://research.ncl.ac.uk/eu-speak/eu-speakbooks/espaol/.

\section{Enfoques, métodos, técnicas y materiales apropiados para alumnos de alfabetización}

Como se ha adelantado, en los siguientes párrafos, vamos a aportar unas notas sobre métodos y recursos útiles en una clase de alfabetización, con énfasis especial en la lectura. En primer lugar, ahondando en las definiciones proporcionadas en los párrafos iniciales, queremos hacer unas precisiones teóricas sobre la lectura (Sosinski 2020):

1. Leer es una actividad que consta de varias habilidades (por ejemplo, conocimiento del idioma, del alfabeto, del contexto cultural).

2. Esas habilidades, metafóricamente, pueden clasificarse en «inferiores» (más automáticas, por ejemplo, reconocimiento de grafemas) y «superiores» (menos automáticas, por ejemplo, reconocimiento del propósito del texto).

3. Los lectores LESLLA deben desarrollar y automatizar las habilidades inferiores para dedicar la atención a las superiores. 
4. El aprendizaje de la lectura debe estar contextualizado y vinculado a la realidad de los alumnos LESLLA.

Por otra parte, en el ámbito de las habilidades inferiores, es imprescindible referirse a dos: la conciencia fonológica y la conciencia grafofonémica.

La conciencia fonológica, simplificando, es el conocimiento explícito de que el lenguaje oral se manifiesta por medios de sonidos y es la base para crear el nexo entre el lenguaje oral y el escrito. La conciencia fonológica es especialmente importante cuando se está aprendiendo a leer en una escritura alfabética. A las personas alfabetizadas les resulta obvio y natural que el discurso se componga de palabras y de otros elementos sucesivos de rango inferior. Si bien separar el discurso en palabras y sílabas es una habilidad universal, no ligada a la alfabetización, no todas las personas no alfabetizadas son conscientes de que hay elementos aún más pequeños, es decir, los fonemas.

Aunque no hay acuerdo sobre si la conciencia fonológica se desarrolla primero y después afecta a la lectura o si esta se desarrolla como resultado de la lectura, es evidente que la conciencia fonémica y la lectura en una escritura alfabética van de la mano -incluso más en el caso de una lengua con una ortografía relativamente transparente, como es el español- (Moats y Tolman 2000), por lo que los materiales para los alumnos de alfabetización deben desarrollar esa habilidad.

El desarrollo de la conciencia fonológica ha sido visto tradicionalmente como un continuum con diferentes grados de dificultad donde podemos identificar tres niveles (Anthony y Lonigan 2004):

1. la conciencia intersilábica, es decir, la conciencia de que las palabras tienen una estructura silábica;

2. la conciencia intrasilábica, o sea, la conciencia de que una sílaba tiene una parte inicial o ataque y una rima o coda;

3. la conciencia fonémica, es decir, la conciencia de que las palabras están formadas a partir de sonidos individuales o fonemas.

Una vez que el alumno haya desarrollado la conciencia fonológica, se puede trabajar la correspondencia sonido/símbolo, la conciencia grafofonémica, es decir, la habilidad de crear nexos entre fonemas y grafemas.

Por último, en la descripción de los métodos nos referiremos a la conocida tipología que diferencia los métodos sintéticos y analíticos (Hernández 2012). El primero, también llamado ascendente, o enfoque fónico o lingüístico, fomenta la enseñanza de las letras o los grafemas y luego de sílabas, palabras y frases. Ese enfoque se centra menos en el significado y más en la conciencia grafofonémica.

El segundo enfoque, el descendente, empieza con frases y palabras. En una clase de ese tipo, solo después del dominio de palabras comunes, los alumnos pasarían al estudio de sílabas, seguido por la manipulación de sonidos y grafemas.

Esta clasificación debe considerarse en paralelo a lo que sabemos sobre las rutas de acceso al texto escrito donde los psicolingüistas suelen diferenciar dos opciones principales (con variaciones y propuestas mixtas a las que no nos referiremos aquí): la ruta léxica (se activa en el caso de palabras conocidas, reconocidas como un todo) y la ruta fonológica (se activa en el caso de palabras nuevas; cada grafema se asocia con un 
fonema, para acceder al procesamiento de unidades superiores) (García Rodríguez y García Díaz 2017).

Asimismo, conviene tener presente la clasificación de Smyser (2016) que diferencia entre los métodos más centrados en la práctica de aspectos lingüísticos y aquellos que ponen énfasis en las funciones sociales de la lectoescritura.

Para terminar esta sección, queremos llamar la atención sobre una tensión metodológica entre los enfoques más comunicativos y más lingüísticos porque, como hemos visto, los resultados de investigación demuestran que las clases de alfabetización efectivas son aquellas que contextualizan la enseñanza y, por otro lado, sabemos que los alumnos se benefician de cursos basados en enfoques fonéticos, más centrados en la habilidad de manipular la forma. Será la tarea del docente encontrar un equilibrio entre esas dos maneras de enseñar.

\subsection{El método de la palabra generadora}

Este método se remonta al trabajo de Paulo Freire en Brasil con adultos no alfabetizados en su lengua materna, el portugués. Freire fue representante de la pedagogía crítica y, por lo que respecta a la alfabetización, de la alfabetización crítica. El proceso debería consistir no solo en la adquisición, o construcción, de ciertas competencias, sino, desde una perspectiva más amplia, en el cambio del rol social de los alumnos que se convierten en participantes activos, capaces de comprender y analizar críticamente la realidad y su propia situación (Freire 1984, 2002).

Observemos que en el pensamiento de Freire se aprecia con claridad que la alfabetización es mucho más que el dominio mecánico del alfabeto. Por otro lado, también se deduce que no tiene sentido alfabetizar en una lengua que se desconoce porque ello impide la construcción del significado (Villalba y Hernández 2000; Miquel 1995). Por último, si los docentes quisieran llevar a la práctica también los postulados políticos y sociales de Freire, y no solo adoptar su metodología de introducir la lectoescritura en la vida de los alumnos, parece un método muy adecuado para inmigrantes adultos no alfabetizados que, en ocasiones, están excluidos de la participación plena en la sociedad.

El trabajo en el aula consiste en la selección de una serie de palabras, las palabras generadoras, a través de un debate del grupo que incluye, en igualdad, al docente y a los alumnos. En el caso del método original, los temas eran más variados; en el caso de los inmigrantes es un momento apropiado para desarrollar la competencia oral y, de acuerdo con la práctica habitual de una clase de lenguas extranjeras, tratar algún núcleo temático relevante para el nivel y los intereses de los alumnos (primeros contactos, vivienda, compra, salud...). Si la competencia oral del grupo es limitada, es relativamente fácil prever qué palabras serán las elegidas para realizar actividades de alfabetización $\mathrm{y}$, probablemente, esas palabras estén también recogidas en los documentos de referencia, como el Plan Curricular del Instituto Cervantes (2006), correspondiente al nivel del grupo.

A continuación, aplicando los procedimientos de análisis y síntesis, se descomponen las palabras en elementos más pequeños, para, a continuación, volver a construir nuevos vocablos. Estas etapas son apropiadas para realizar una variedad de actividades con el fin de desarrollar en los alumnos la conciencia fónica, para lo cual existen baterías de 
actividades, sobre todo dirigidas a niños, pero fácilmente adaptables a adultos. De esta forma, paulatinamente, se introduce el alfabeto completo. A continuación (Imagen 1) se muestra una foto de una clase basada en el método de Freire y que trata el tema de la vivienda. Se puede observar la descomposición de palabras en sílabas.

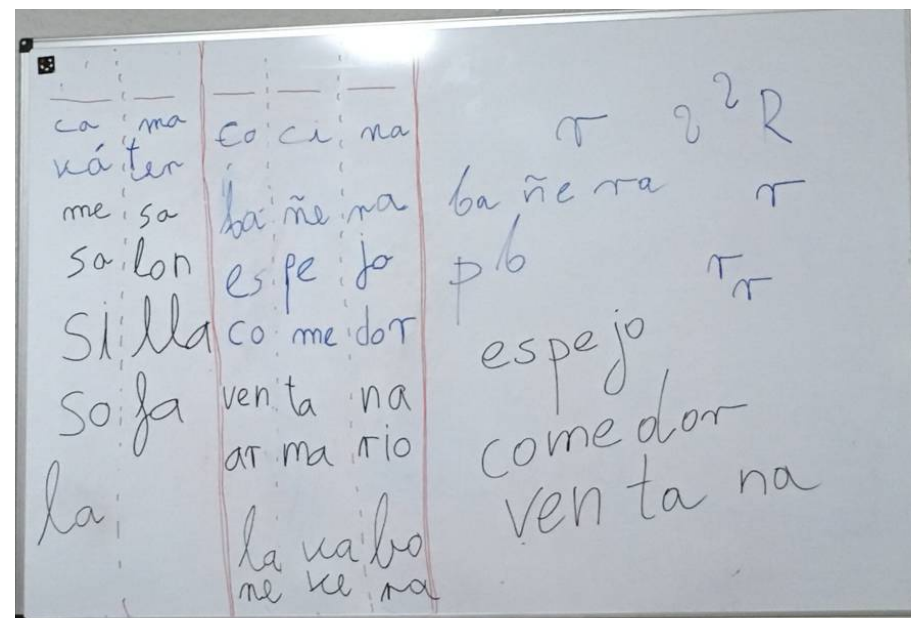

Imagen 1. La pizarra en una clase basada en el método de Freire

La dificultad de aplicación de ese método aparece en las últimas etapas porque los alumnos inmigrantes no tienen la competencia léxica de un nativo y, por lo tanto, con frecuencia no son capaces de crear elementos nuevos, a partir de los grafemas y las sílabas trabajados en clase.

En resumen, es un método analítico mitigado (Hernández 2012) que le concede mucha importancia al desarrollo de la conciencia fonológica a través de una variedad de ejercicios mecánicos (Smyser 2016), pero también permite trabajar diferentes aspectos de la competencia oral y de la vida social de la comunidad receptora. Para ver su aplicación práctica, se pueden revisar los recursos del Instituto Estatal de la Educación para los Adultos (http://www.inea.gob.mx/index.php), su método Pala y los vídeos de demostración (https://www.youtube.com/channel/UC-5iQQ-jpFrDUXUDZb3vVuw).

En general, es un buen ejemplo de un método analítico para alumnos de niveles bajos y se sitúa en la misma órbita que los conocidos manuales como Contrastes (Colón et al. 1999) o Oralpha (Vilar 2008) que, sin seguir sus principios, también adoptan el enfoque descendente.

\subsection{EI Instructor Digital de Alfabetización}

Uno de los efectos de la creación de la asociación LESLLA fue el aumento de la actividad de redes internacionales, no solo entre los investigadores, sino también entre los profesionales. Dado que el foro se gestó en los Países Bajos, con el simposio inaugural en Tilburg, parecía natural establecer lazos con profesionales fuera del mundo anglosajón. Un ejemplo de esa colaboración fue un proyecto europeo que dio como fruto la herramienta Instructor Digital de Alfabetización ${ }^{2}$, en el que participaron cuatro países (Países Bajos, Alemania, Reino Unido y Finlandia). DigLin ahora también está

\footnotetext{
${ }^{2}$ Visite https://www.nt2.nl/en/diglin; la versión española: https://test.fcsprint2.nl/menu/24
} 
disponible en danés, francés y español (para descripciones de DigLin e informes sobre sus pruebas iniciales, véase Cucchiarini et al. 2013; Cucchiarini et al. 2015; Naeb y Sosinski 2020a; Naeb y Sosinski 2020b).

DigLin es una plataforma digital que introduce al alumno en la correspondencia básica grafema-fonema, utilizando palabras significativas para un inmigrante adulto en un idioma y, en los últimos desarrollos, también frases, oraciones y textos más largos. El primer paso fue la creación de una barra de sonido como herramienta para dar al usuario una visión general de todo el alfabeto de un idioma dado, con sus grafemas simples, dígrafos y trígrafos. Los usuarios escuchan los sonidos correspondientes a cada grafema, dígrafo o trígrafo y comienzan a establecer las conexiones letra/grafemasonido/fonema. El segundo paso consistió en recopilar una lista de 300 palabras reales y útiles para inmigrantes adultos, que fueran sencillas en su construcción fonológica, que representaran diferentes posibilidades ortográficas de la lengua en cuestión y que pudieran representarse con facilidad mediante fotografías. Por último, el tercer paso fue la creación de 15 juegos de ejercicios para cada idioma. Cada bloque tiene las siguientes actividades que incluyen, por ejemplo, arrastrar y soltar grafemas o escribir respuestas, y pueden ir acompañadas de audio y fotografías: 1) introducción del vocabulario; 2) relación fonemas/grafemas; 3) actividades con palabras enteras; 4) trabajo con secuencias de fonemas/grafemas; 5) automatización de las correspondencias grafemafonema.

Es importante señalar que DigLin se basa en el método educativo de FC Sprint2, desarrollado por uno de los socios holandeses en el Friesland College (véase Deutekom 2008) y en el concepto de altas expectativas. DigLin proporciona al alumno una exposición extensa pero individualizada al input, acompañada de una retroalimentación inmediata. Se centra en el alumno y el control del aprendizaje pasa del profesor - cuyo papel se convierte en el de un facilitador - al alumno que decide cómo realizar las actividades. Los alumnos pueden utilizar el programa informático como deseen, solos o con otros alumnos y con la ayuda del profesor, si así lo deciden. DigLin, que puede utilizarse en diversos dispositivos digitales, incluidos tabletas y teléfonos móviles, no tiene por qué utilizarse en el aula, pero cuando se hace, el docente alentará a los alumnos a utilizar el programa informático de diversas maneras. Por ejemplo, en el nivel inicial, el profesor puede pedir a los alumnos que encuentren varias palabras sobre alimentos que empiecen con la letra $<_{\mathrm{s}}>$. Los alumnos utilizan entonces sus propias estrategias para navegar por la plataforma y averiguar cómo leer las palabras (Naeb y Sosinski 2020a, 2020b; Young-Scholten, Naeb y Sosinski 2020).

Los estudios de campo realizados en cada país durante el proyecto original y dos estudios de seguimiento (Filimban 2019; Malessa y Filimban 2017) muestran un notable progreso en la decodificación de palabras por parte de los alumnos que utilizan Diglin. Los resultados también revelan que los alumnos aumentaron la autonomía, desarrollaron estrategias de aprendizaje, aumentó su motivación y demostraron un alto nivel de satisfacción con el programa (van de Craats y Young-Scholten 2015).

Por último, esta herramienta es un ejemplo de métodos que conceden mucha importancia a las actividades de corte lingüístico, en la línea de lo que señalaban Kurvers y van der Zouw (1990) y nos parece especialmente útil para el trabajo autónomo del alumno. En cambio, las sesiones presenciales pueden destinarse a otras actividades de orientación más social. 


\subsection{El enfoque de la experiencia lingüística}

Este enfoque aborda la decodificación y la comprensión lectora de manera inicialmente descendente, como el método de la palabra generadora, pero el punto de partida es el texto. Además, las bases de este método son similares al método de la palabra generadora, aunque, en un principio, no se trata de una metodología tan fuertemente ideologizada.

En este caso, los alumnos, con o sin ayuda del profesor, construyen un texto y lo escriben en un lugar visible de la clase (Hall 1970; Taylor 1992). El tema ideal es una experiencia común que, si es necesario, se puede crear: un paseo por la ciudad, una película o una comida compartida o preparada en grupo. Si los alumnos no tienen suficiente nivel para escribir de forma autónoma, el profesor se encarga de escribir el texto, pero no de modificarlo ni corregirlo: tan solo se limita a ejercer el papel de escriba, si bien, más tarde, ese texto podrá ser enmendado. A continuación, se pueden realizar actividades de análisis y síntesis que centran la atención del grupo en los aspectos lingüísticos. En función del nivel del grupo, esas actividades pueden centrarse en habilidades inferiores (grupos principiantes) o superiores (grupos más avanzados) relacionadas con la lectoescritura. Las segundas, que no hemos tratado en detalle en este texto, son actividades habituales en clases de idiomas de alumnos alfabetizados que aprenden a crear textos en la lengua meta (Cassany et al. 1994; Cassany 2004).

Se trata de un método analítico (Hernández 2012) que prioriza el significado y las funciones sociales de la escritura (Smyser 2016)

A continuación (Imagen 2), como ejemplo, pueden observar el texto creado por los alumnos de una profesora que participó en los cursos de formación de EU-SPEAK.

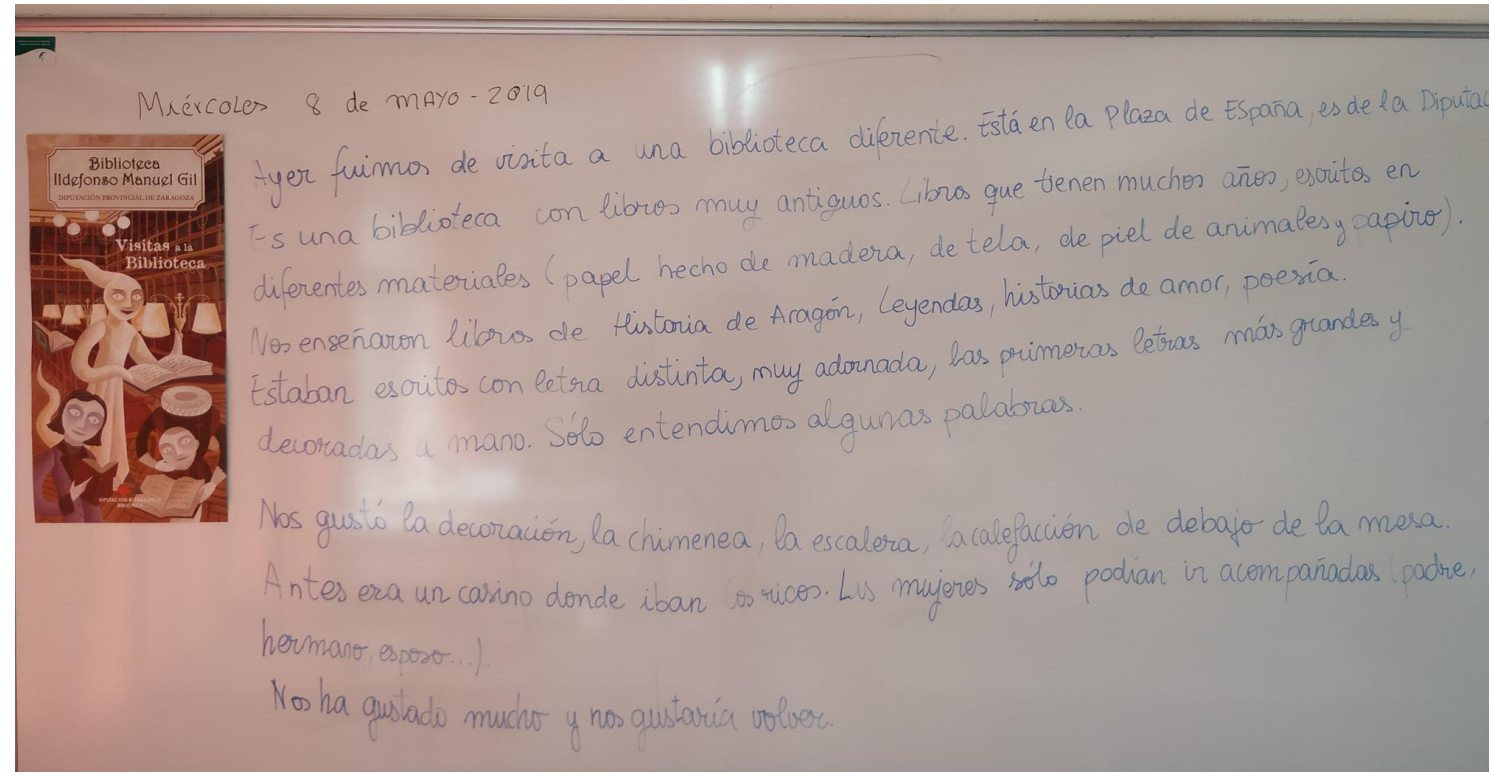

Imagen 2. Texto creado en una clase basada en el método enfoque de experiencia lingüística 


\subsection{La lectura extensiva}

Mientras que la decodificación/reconocimiento de palabras en el nivel inferior depende principalmente del dominio de la fonología del nuevo idioma, la comprensión de las frases depende de la competencia lingüística, en sus vertientes gramatical y léxica. La comprensión de secuencias más largas supone dominar la integración palabra-texto que se irá automatizando poco a poco para liberar la capacidad de atención necesaria, a su vez, para comprender un texto compuesto de varias oraciones (Grabe 2004, 2009; Koda 2005; Kuhn y Stahl 2003; Rasinski 2003; Segalowitz 2010).

A ese respecto, hay numerosas evidencias (por ejemplo, el informe PISA de 2002) de que hay una actividad en la que participan niños, adolescentes y adultos de todo el mundo, independientemente de su situación socioeconómica, y que es fundamental para alcanzar niveles más altos de comprensión lectora. Esta práctica se denomina, por ejemplo, lectura extensiva, lectura de placer, lectura voluntaria libre, etc. Durante su ejercicio, el individuo lee lo que quiere, cuando, donde y como quiere. En el caso de los estudiantes de un segundo idioma de todas las edades y niveles de competencia, la lectura extensiva se correlaciona positivamente con el aumento del vocabulario y de la complejidad morfosintáctica de su interlengua (por ejemplo, Bamford y Day 2004; Coady 1997; Horst 2005; Krashen 1989; Pigada y Schmitt 2006). Hay una base común entre la lectura por placer y el enfoque de experiencia lingüística, en el sentido de que los dos se basan en las experiencias, expectativas y capacidades de inferencia del individuo para fomentar la alfabetización crítica (Friere 2002) que a menudo está ausente en las aulas que atienden a los adultos inmigrantes (Cardiff et al. 2007; Duncan 2014; Graff 1993). Pero a diferencia del otro método, la lectura libre no es dirigida por el profesor, sino por el propio alumno. En cuanto a la temática de los textos, los alumnos parecen preferir la ficción general, seguida de las autobiografías (Rodrigo et al. 2007). La lectura de narraciones parece aumentar la asimilación de aspectos lingüísticos del texto (Lee 2009) y aumenta la autonomía del alumno, así como causa un mayor bienestar general (Dijikic et al. 2009; Mar y Oatley 2008). Actualmente hay un número reducido de estudios a pequeña escala sobre los alumnos adultos cuyo nivel del dominio de una lengua se sitúa por debajo de A1, según el MCER. Sus autores describen los beneficios similares a los de muchos otros estudios sobre la lectura: aumenta la competencia lingüística, el pensamiento crítico y también la motivación para leer más (incluso con los niños en la familia) y para integrarse en las actividades de la comunidad (Constantino 1995; Laymon 2013, 2015; Rodrigo et al. 2007; Williamson 2013; Yaden et al. 2003).

Lamentablemente, apenas hay libros de ficción para los estudiantes sin alfabetizar, es decir, para los que todavía aprenden a decodificar las palabras, con un nivel de competencia léxica y gramatical bajo. Del estudio de Rodrigo et al. (2007), recogemos la recomendación de aproximadamente cuántos libros se requieren para una clase con estudiantes LESLLA. En su programa se utilizó una proporción de 6:1, es decir, por cada estudiante de nivel intermedio, había seis libros. Aplicando esa proporción, un programa de lectura extensivo para una clase de 15 estudiantes de alfabetización requeriría 90 libros.

Young-Scholten (2017) concluyó que hay entre 100 y 200 de esos libros en inglés de varias editoriales. Es un número muy pequeño en comparación con los millones de 
libros en todo el mundo, tanto en inglés como en otros idiomas, escritos para adultos formados y para niños pequeños. La cantidad de libros para los estudiantes de alfabetización es quizás suficiente para un año y para que una clase determinada se dedique a la lectura extensiva. Para organizar un programa de ese tipo, es necesario reservar un tiempo para la lectura silenciosa y dar a los alumnos la oportunidad de leer libros fuera del aula, por ejemplo, facilitando el préstamo de libros en papel o el acceso a libros electrónicos en dispositivos digitales, incluso en teléfonos móviles. El papel del profesor es inculcar al alumno el hábito de leer por placer, es decir, motivarlo y no controlar qué leen los alumnos.

Como hemos dicho, es necesario que haya suficientes libros para que los lectores se aficionen a la lectura, pero la escasez de recursos hace que la lectura extensiva con ese grupo de estudiantes sea poco frecuente. Es un desafío abordar esa escasez de recursos, ya que no hay un gran mercado comercial para tales libros. Durante la última década, la iniciativa Simply Cracking Good Stories, con la participación de estudiantes de Lengua Española/Inglesa y Lingüística, bajo la supervisión de dos autores de este artículo, en las universidades de Granada y Newcastle (véase Young-Scholten et al. 2017), ha comenzado a llenar el vacío de recursos, produciendo libros y distribuyéndolos gratuitamente. La iniciativa Simply Cracking Good Stories aplica los siguientes criterios en la creación de los libros: (1) no más de 400 palabras; (2) imágenes para realzar el texto, pero no para contar la historia; (3) vocabulario limitado y sintaxis simple; (4) tramas narrativas de interés para cualquier adulto, no solo para los inmigrantes, que apliquen los principios de la escritura creativa de ficción (véase, por ejemplo, Toolan 2009).

El último punto ha sido muy problemático porque los escritores corren el riesgo de adoptar una actitud paternalista y asumir que los adultos inmigrantes analfabetos son diferentes en cuanto a los temas que les interesan. Sin embargo, si un adulto nunca ha leído un libro en su vida, no se puede anticipar qué tipo de temas le gustarán. Fundamental para el éxito de la lectura extensiva es la libertad del lector para decidir qué leer (Murphy 1987) y esa decisión, a veces, puede significar la elección de un tema tabú o peligroso. Es importante reconocer que el interés por esos temas es típico de los adultos; basta con considerar unos de los géneros más populares de los adultos alfabetizados: la ficción de crimen, de suspense o de terror. Por supuesto, los libros sobre temas tabú o perturbadores son difíciles, si no imposibles, de leer en grupo y en voz alta, pero son fáciles de disfrutar como actividad individual. Descubrir lo que a los estudiantes les gusta leer y responder a sus preferencias con más libros, así como observar sus reacciones son los objetivos actuales del mencionado proyecto. Además, en el caso de Newcastle, en los últimos tiempos, se ha contado con la orientación y ayuda de escritores creativos y de editoriales profesionales.

Según los criterios citados al comienzo de esta sección, se trata de un método descendente y orientado hacia la exploración del valor social de la lectoescritura.

\subsection{La alfabetización en lenguas de herencia}

$\mathrm{Si}$, como se ha dicho, es posible transferir la alfabetización del idioma nativo, ¿no tendría sentido que un adulto no alfabetizado dominara primero la lectura en ese idioma? Es lo que una nueva iniciativa, el Heritage Language Hub (Plataforma de 
Lenguas de Herencia), se propone conseguir. El equipo que lo compone es pequeño y actualmente incluye académicos y profesionales de Canadá, Italia, Turquía, los Estados Unidos y el Reino Unido. La misión del HL Hub es proporcionar acceso a recursos para la alfabetización en las lenguas maternas de los inmigrantes. Además, el objetivo de la Plataforma es poner en valor y fomentar el mantenimiento en la comunidad de las lenguas maternas de los alumnos LESLLA, para asegurarse de que sus niños tengan la oportunidad de crecer bilingües y alfabetizados, tanto en ese idioma como en el idioma mayoritario, que es el idioma de la escolarización (véase Cummins 2000a, b; Cummins y Danesi 1990; Fishman 2004; Polinsky y Kagan 2007; Ruiz 1984; Shin 2013). De hecho, las investigaciones demuestran que la educación en el idioma de herencia de los niños les proporciona el mejor punto de partida para tener los mejores resultados finales del proceso educativo (Baker y Wright 2017; Carlo y Skilton-Sylvester 1996; Cummins 1979, 1992; Cummins y Swain 1986; Harju-Luukkainen et al. 2014; Kenner 2004; Leikin et al. 2010; Schwartz et al. 2007; Thomas y Collier 1997; Wagner y Venezky 1999).

Además, es una iniciativa que responde a un cambio entre los educadores de adultos, que han pasado de centrarse en la integración en la lengua mayoritaria y su cultura para participar eficazmente en la vida social, educativa y económica del país en el que se han reasentado los inmigrantes (Bigelow 2009; Cummins 2000a, b, 2005; Duff 2001; Mallows 2014; Simpson y Whiteside 2015) - a apoyar las lenguas propias de los alumnos. Es lo que ha ocurrido ya en el caso de los grupos de inmigrantes más instruidos, pero mucho menos en el caso de los grupos de inmigrantes con niveles bajos de instrucción formal. Los profesionales y responsables de cursos tienden a descuidar las lenguas que hablan los inmigrantes, especialmente las lenguas que hablan los adultos no alfabetizados (Aberdeen 2016; Peyton 2012, 2013).

El primer paso para el equipo de la Plataforma de Lenguas de Herencia fue la creación de la plataforma, con enlaces que luego dan acceso a cientos de recursos digitales, es decir, a libros, archivos de audio y materiales para los profesionales. Actualmente, la gran mayoría son enlaces a recursos para niños y los sitios web están en inglés. El equipo está trabajando en la identificación de recursos en otros idiomas de los diversos países de los que proviene y en los que se reubican los estudiantes LESLLA, al mismo tiempo que se están explorando formas de proporcionar libros escritos para adultos más allá de las lenguas de herencia principales en Estados Unidos, el árabe y el español (para obtener más información, visite: https://www.leslla.org/resources-in-mother-tongues).

El equipo es consciente de que los profesionales que trabajan con los estudiantes no alfabetizados no están preparados para incorporar las lenguas de herencia en sus aulas, por lo que, además de crear un banco de recursos, se han dirigido grupos de discusión y se han comenzado a desarrollar ideas y establecer protocolos para hacerlo con éxito.

Por ejemplo, una técnica, relacionada con la lectura libre, consiste en que los alumnos adultos trabajan con materiales de lectura en principio destinados a sus niños y/o a los jóvenes de sus propias comunidades. Los alumnos discuten los libros, comparten datos sobre los diversos idiomas en los que están escritos los libros y cuentan las historias que contiene. Esta actividad también supone una reflexión colectiva y compartida sobre el lenguaje como objeto y aumenta la conciencia de las características gramaticales, socioculturales y pragmáticas de las muchas lenguas que hablan los estudiantes de un grupo determinado (Beacco et al. 2016 Pugliese y Malavolta 2017). 
Esta propuesta, que guarda similitudes con la lectura extensiva, apuesta por un enfoque descendente en el aprendizaje de alfabetización $\mathrm{y}$, como se ha podido observar, tiene un importante componente social.

\section{Recapitulación}

El objetivo de este artículo era ofrecer unas notas sobre enfoques, métodos y recursos que los profesores de nuestro ámbito cultural utilizan en las clases de alfabetización para inmigrantes adultos. Además, queríamos hacerlo desde una perspectiva teóricopráctica, apoyando nuestras reflexiones en resultados de investigación contrastados.

En primer lugar, precisamos los términos y diferenciamos varios matices dentro del término no alfabetizado. Luego realizamos unas observaciones sobre las similitudes y diferencias entre los inmigrantes adultos no alfabetizados, los niños y los adultos no nativos, pero alfabetizados. Finalmente aludimos a los resultados de investigación sobre qué tipo de instrucción es la más efectiva.

Sobre esa base hemos caracterizado cinco métodos y recursos: el método de la palabra generadora, el Instructor Digital de Alfabetización, el enfoque de experiencia lingüística, la creación de libros para la lectura libre y el centro de lenguas de herencia. Todos están pensados específicamente para alumnos de alfabetización, si bien su aplicación dependerá del perfil del grupo y del nivel de la competencia lectoescritora alcanzado.

Queremos cerrar este trabajo proclamándonos partidarios de una metodología equilibrada, descendente (para incluir el significado desde el primer momento) y ascendente (para no obviar la necesidad de desarrollar la conciencia fonológica de los alumnos). Igualmente, invitamos a los lectores a participar en la creación de materiales de lectura para el colectivo de adultos no alfabetizados, a ponerse en contacto con los miembros de la Plataforma de Lenguas de Herencia para ampliar la lista de recursos disponibles y a participar en las actividades de la asociación LESLLA.

\section{Bibliografía:}

ABERDEen, T. (2016). Understanding Community Heritage Language Schools in Alberta. Tesis doctoral. University of Alberta, Canada.

AlAMPRESE. J. (2009). «Developing Learners' Reading Skills in Adult Basic Education Programs». En S. Reder y J. Bynner. (eds.), Tracking Adult Literacy and Numeracy Skills (pp. 107-131). Londres: Routledge.

Anthony, J. y Lonigan, C. (2004). «The Nature of Phonological Awareness: Converging Evidence from Four Studies of Preschool and Early Grade School Children». Journal of Educational Psychology, 96 (1), pp. 43-55.

BAKER, C., y Wright, W. E. (2017). Foundations of Bilingual Education and Bilingualism. Clevedon, UK: Multilingual Matters.

BAMFORD, J. y DAY, R. (Eds.). (2004). Extensive Reading Activities for Teaching Language. Cambridge: CUP. 
Beacco, J.-C., Byram, M., Cavalli, M., Coste, D., Cuenat, M., Goullier, F., y PANTHIER, J. (2016). Guide for the Development and Implementation of Curricula for Plurilingual and Intercultural Education. Recuperado de: http://www.coe.int/t/DG4/linguistic/Source/Source2010_ForumGeneva/GuideEPI2010 EN.pdf

Becker, A., Dittmar, N., Gutmann, M., Klein, W., Rieck, B-O., Senft, G., Senft, I., STECKNER, W. y THIELICKE, E. (1977). Heidelberger Forschungsprojekt "PidginDeutsch spanischer und italienischer Arbeiter in der Bundesrepublik": Die ungesteurerte Erlernung des Deutschen durch spanische und italienischer Arbeiter. Eine soziolinguistische Untersuchung. [Heidelberg Research Project 'The Pidgin German of Spanish and Italian Workers in West Germany': The Uninstructed Acquisition of German by Spanish and Italian Workers: A Sociolinguistic Study]. Ostabrück: Osnabrücker Beiträge zur Sprachtheorie.

Bigelow, M. (2009). «Social and Cultural Capital at School: The Case of a Somali Teenage Girl». Low-educated Second Language and Literacy Acquisition: Research, Policy, and Practice: Proceedings of the Second Annual Forum (pp. 7-22). Richmond, VA: The Literacy Institute, Virginia Commonwealth University.

Burt, M., Peyton, J.K., y Schaetzel, K. (2008). Working with Adult English Language Learners with Limited Literacy: Research, Practice, and Professional Development. Center for Adult English Language Acquisition.

Cardiff, P., Newman, K., y Pearce, E. (2007). Reflect for ESOL Resource Pack. ActionAid. Recuperado de:

http://www.actionaid.org.uk/doc lib/reflect for esol resource_pack.pdf

CARlo, M. S., y Skilton-Sylvester, E. E. (1996). A Longitudinal Investigation on the Literacy Development of Spanish-, Korean-, and Cambodian-Speaking Adults Learning to Read English as a Second Language. Manuscrito no publicado. Philadelphia, PA: National Center on Adult Literacy.

CAssany, D., LunA, M. y SAnZ, G. (1994). Enseñar lengua. Barcelona: Graó.

CASSANY, D. (2004). La cocina de la escritura. Barcelona: Anagrama.

Chall, J. S. (1999). «Models of Reading». En D. A. Wagner, R. L. Venezky y B. Street. (Eds.), Literacy: An International Handbook (pp. 163-166). Nueva York: Garland.

Clahsen, H., Meisel, J. y Pienemann, M. (1983). Deutsch als Zweitsprache: Der Spracherwerb ausländischer Arbeiter. Tubinga: Gunter Narr.

COADY, J. (1997). «L2 Vocabulary Acquisition Through Extensive Reading». En J. Coady y T. Huckin. (eds.), Second Language Vocabulary Acquisition (pp. 225-237). Cambridge: CUP.

Colón, M. ${ }^{a}$ J., Julve, M., Montaner, P., Orgilés, M. ${ }^{a}$ P., y SÁez, T. (1999). Método Contrastes. Alfabetización en español como lengua extranjera. Madrid: Ministerio de Educación y Cultura.

Condelli, L., Spruck Wrigley, H., Yoo, K., Seburn, M., y Cronen, S. (2003). What Works. Study for Adult ESL Literacy Students. Volume II: Final Report. Washington, DC: US Department of Education. 
Constantino, R. (1995). «Learning to Read in a Second Language Doesn't Have to Hurt: The Effect of Pleasure Reading», Journal of Adolescent and Adult Literacy, 39, pp. 68-69.

Cucchiarini, C., van de CraAts, I., DeuteKom, J., y StRiK, H. (2013). «The Digital Instructor for Literacy Learning». En B. Badlin, T. Hueber, G. Bailly, D. Demolin y F. Raby (eds.), Proceedings of the SLaTE-2013 workshop (pp. 96-101).

Cucchiarini, C., Dawidowicz, M., Filimban, E., Tammelin-Laine, T., Van de CRAATS, I. y STRIK, H. (2015). «The Digital Literacy Instructor: Developing Automatic Speech Recognition and Selecting Learning Material for Opaque and Transparent Orthographies». En I. van de Craats, J. Kurvers y R. van Hout. (eds.), Adult literacy, Second Language and Cognition (pp. 251-278). Nijmegen: CLS.

Cummins, J. (1979). «Linguistic Interdependence and the Educational Development of Bilingual Children», Review of Educational Research, 49 (2), pp. 222-251.

Cummins, J. (1992). «Heritage Language Teaching in Canadian Schools», Journal of Curriculum Studies, 24 (3), pp. 281-286.

Cummins, J. (2000a). Language, Power, and Pedagogy: Bilingual Children in the Crossfire. Clevedon, UK: Multilingual Matters.

Cummins, J. (2000b). «Putting Language Proficiency in its Place: Responding to Critiques of the Conversational /Academic Language Distinction». En J. Cenoz y U. Jessner (eds.), English in Europe: The Acquisition of a Third Language (pp. 54-83). Clevedon, UK: Multilingual Matters.

Cummins, J. (2005). «A Proposal for Action: Strategies for Recognizing Heritage Language Competence as a Learning Resource within the Mainstream Classroom». The Modern Language Journal, 89 (4), pp. 585-592.

Cummins J., y DAnesi, M. (1990). Heritage Languages: The Development and Denial of Canada's Linguistic Resources. Montreal, Canada: Our Selves Education Foundation.

DeuTEKom, J. (2008). FC-Sprint ${ }^{2}$ : Grenzeloos leren. Boom.

Djikic, M., Oatley, K., Zoeterman, S., y Peterson, J. B. (2009). «On Being Moved by Art: How Reading Fiction Transforms the Self». Creativity Research Journal, 21 (1), pp. 24-29.

DunCAN, S. (2014). Reading for Pleasure and Reading Circles for Adult Emergent Readers. Leicester: National Institute for Adult Continuing Education.

DuRgunOĞLU, A.Y. (2012). «Adult Literacy and Empowerment: Description and Evaluation of a Program in Turkey». En P. Vinogradov y M. Bigelow. (eds), Proceedings of the 7th Low Educated Second Language and Literacy Acquisition Symposium (pp. 27-46). Minneapolis: Universidad de Minnesota.

DufF, P. (2001). «Language, Literacy, Content, and (Pop) Culture: Challenges for ESL Students in Mainstream Courses», The Canadian Modern Language Review/La Revue Canadienne de Langue Vivantes, 58(1), pp. 103-132. 
EHRI, L. (1994). «Development of the Ability to Read Words. Update». En R. Ruddell, M. Ruddell y H. Singer. (eds.), Theoretical Models and Processes of Reading (pp. 323358). Newark, DE: IRA.

Filimban, E. (2019). The Effectiveness of a Computer Software for Developing Phonemic Awareness and Decoding Skills for Low-literate adult learners learning English. Tesis doctoral. Universidad de Newcastle.

Fishman, J. A. (2004). «Language Maintenance, Language Shift, and Reversing Language Shift». En T. K. Bhatia y W. C. Ritchie (eds.), The Jandbook of Bilingualism (pp. 406-436). Oxford, UK: Blackwell Publishing.

FreIRE, P. (1984, 9a ed.). Pedagogía del oprimido. Madrid: Siglo XXI de España Editores.

FreIRE, P. (2002, $11^{\mathrm{a}}$ ed.). La educación como práctica de la libertad. Madrid: Siglo XXI.

GARCÍA RodríGUEZ, M. L. y GARCÍA DÍAZ, N. (2017). «Cincuenta palabras generatrices para el aprendizaje del código escrito de la lengua española. Adaptación del método de Paulo Freire a la edad infantil», Didáctica Lengua y Literatura, 29, pp. 81-102.

Goswami, U. y Bryant, P. E. (1990). Phonological Skills and Learning to Read. Hove: Psychological Press.

GRABE, W. (2004). «Research on Teaching Reading», Annual Review of Applied Linguistics, 24, pp. 44-69.

GRABE, W. (2009). Reading in a Second Language. Cambridge: CUP.

GrafF, G. (1993). Beyond the Culture Wars. Nueva York: Norton.

Hall, M. A. (1970). Teaching Reading as a Language Experience. Columbus, OH: Charles Merrill.

Harju-Luukkainen, H., Nissinen, K., Sulkunen, S., Suni, M., y Vettenranta, J. (2014). Avaimet osaamiseen ja tulevaisuuteen. Selvitys maahanmuuttajataustaisten nuorten osaamisesta ja siihen liittyvistä taustatekijöistä PISA 2012-tutkimuksessa. [Keys to competence and a future. A Report on PISA 2012 results and related underlying factors for students with an immigrant background]. Jyväskylä, Finland: University of Jyväskylä, Finnish Institute for Educational Research.

HAWKIns, R. (2001). Second Language Syntax: A Generative Introduction. Oxford: Blackwell.

HERnÁNDEZ GARCÍA, M. ${ }^{\mathrm{a}}$ T. (2012). Aprendizaje de la lectoescritura en una segunda lengua por parte de adultos analfabetos en su LM. Una propuesta de alfabetización en español como segunda lengua (L2). Memoria de doctorado DEA. Alicante: Universidad de Alicante.

Horst, M. (2005). «Learning L2 Vocabulary through Extensive Reading: A Measurement Study». Canadian Modern Language Review, 61, pp. 355-382.

Instituto Cervantes (2006). Plan curricular del Instituto Cervantes. Madrid: Instituto Cervantes. 
JoHnsOn T. y BERRY, S. (2014). «Beyond the ESOL Classroom». En D. Mallows. (ed.), Language Issues in Migration and Integration: Perspectives from Teachers and Learners (pp. 81-94). Londres: British Council.

Juel, C. (1991). «Beginning Reading». En P. D. Pearson, R. Barr, M. L. Kamil. (eds.), Handbook of Reading Research (pp. 759-787). London: Longman.

KENNER, C. (2004). «Living in Simultaneous Worlds: Difference and Integration in Bilingual Script-Learning», International Journal of Bilingual Education and Bilingualism, 7 (1), pp. 43-61.

KLEIN, W. y PERDUE, C. (1997). «The Basic Variety (or: Couldn't Natural Languages be Much Simpler?)», Second Language Research, 13, pp. 301-347.

KodA, K. (2005). Insights into Second Language reading: A Cross-Linguistic Approach. Cambridge: Cambridge University Press.

KrASHEN, S. (1985). The Input Hypothesis. Issues and Implications. London: Longman.

KRASHEN, S. (1989). «We Acquire Vocabulary and Spelling by Reading: Additional Evidence for the Input Hypothesis». Modern Language Journal, 73, pp. 440-64.

Kuhn, M. R. y Stahl, S. A. (2003). «Fluency: A Review of Developmental and Remedial Practices», Journal of Educational Psychology, 95 (1), pp. 3-21.

KurVERS, J. (2002). Met Ongeletterde Ogen. Kennis van taal en schrift van analfabeten. Ámsteram: Akskant.

KURVERS, J., y STOCKMANN, W. (2009). «Alfabetisering NT2 in beeld. Leerlast en succesfactoren». Report. Tilburg University.

Kurvers, J., StockmanN, W. y VAN DE CRAATS, I. (2010). «Predictors of Success in Adult L2 Literacy Acquisition». En T. Wall y M. Leong. (eds.), Low Educated Second Language and Literacy Acquisition (pp. 47-62). Calgary: Bow Valley College.

KURVERS, J. y VAN DER ZOUW., K. (1990). In de ban van het schrift. Over analfabetisme en alfabetisering in een tweede taal. (Charmed by Writing. About Illiteracy and Literacy Acquisition in a Second Language). Amsterdam: Swets and Zeitlinger.

LAymon, L. (2013). Case study: A Model of an Extensive Reading Programme Inside an ESL Low-Level Classroom. Comunicación presentada en LESLLA 2013, San Francisco.

LAYMON, L. (2015). «Extensive Reading in Low-Level ESL: Can It be Done?». En M. G. Santos y A. Whiteside. (eds.). Low Educated Second Language and Literacy Acquisition. Proceeding from the Ninth Symposium. San Francisco: Lulu Publishing Services.

LEE, S-K. (2009). «Topic Congruence and Topic of Interest: How do They Affect Second Language Reading Comprehension?», Reading in a Foreign Language, 21, pp. 159-178.

LeIKIN, M., Schwartz, M., y Share, D. L. (2010). «General and Specific Benefits of Bi-literate Bilingualism: A Russian-Hebrew Study of Beginning Literacy», Reading and Writing, 23, pp. 269-272. 
LENNEBERG, E. H. (1967). Fundamentos biológicos del lenguaje. Madrid: Alianza Editorial, 1975.

Malessa, E. y Filimban, E. (2017). Exploring what Log Files Can Reveal about LESLLA Learners' Behaviour in an Online CALL Environment. En M. Sosiński. (Ed.). Language and Literacy. Teaching LESLLA Students. Granada: Universidad de Granada.

Mallows, D. (Ed.) (2014). Language Issues in Migration and Integration: Perspectives from Teachers and Learners. London, UK: The British Council.

Manifiesto de Santander (2004). Recuperado de: https://cvc.cervantes.es/ensenanza/biblioteca_ele/inmigracion/documentos/manifiesto.ht $\mathrm{m}$

Mar, R. A. y OAtley, K. (2008). "The Function of Fiction is the Abstraction and Simulation of Social Experience», Perspectives on Psychological Science, 3 (3), pp. 173-192.

Morais, J., Content, A., Bertelson, P., CARy, L. y Kolinsky, R. (1988) «Is There a Critical Period for the Acquisition of Segmental Analysis?», Cognitive Neuropsychology, 5, (3), pp. 347-352.

Miquel LóPEZ, L. (1995). «Reflexiones previas sobre la enseñanza de E/LE a inmigrantes y refugiados», Didáctica. Lengua y literatura, (7), pp. 241-254.

Murphy, B. M. Z. (1987). «Bad Books in Easy English», Modern English Teacher, 14 (3), pp. 22-23.

NAEB, R., y Sosinski, M. (2020a). «Technology-Enhaced Learning (TEL) in the LESLLA Context / Aprendizaje potenciado por la tecnología (TEL) en el contexto LESLLA». En M. Planelles Aleida, J. Muñoz Liceras y A. Foucart. (eds.), Perspectivas actuales en la enseñanza y el aprendizaje de lenguas en contextos multiculturales / Current Perspectives in Language Teaching and Learning in Multicultural Contexts, Thomson Reuters: Aranzadi.

NAEB, R., y Sosinski, M. (2020b). «De los sonidos a las palabras: el aprendizaje asistido por tecnología para desarrollar la lectura de migrantes adultos». En N. Gómez López y J. M. Fernández Campoy. (eds.), Las metodologías didácticas innovadoras como estrategia para afrontar los desafios educativos del siglo XXI (pp. 239-255). Madrid: Dykinson.

Olson, D. R. y Torrance, N. (Eds.). (2009). The Cambridge Handbook of Literacy. Cambridge: CUP.

Perfetti, C. A. (2010). «Decoding, Vocabulary and Comprehension: The Golden Triangle of Reading Skill». En M. G. McKeown y L. Kucan. (eds.), Bringing Reading Research to Life: Essays in Honor of Isabel Beck (pp. 291-303). Nueva York: Guilford.

Peyton, J. K. (2012). «Understanding Adult Learners as Multilingual/Multicultural Individuals: Practical and Research Implications». En M. Bigelow y P. Vinogradov (Eds.), Proceedings from the $7^{\text {th }}$ Annual LESLLA (Low Literate Second Language and Literacy Acquisition) Symposium. Minneapolis, MN: University of Minnesota.

Peyton, J. K. (2013, August). «Recognizing, Valuing, and Building on Heritage Cultures and Languages in English Language Programs». Bilingual Basics: Newsletter of the Bilingual Education Interest Section and Teachers of English to Deaf Students. 
Teachers of English to Speakers of Other Languages. Recuperado de: http://newsmanager.commpartners.com/tesolbeis/issues/2013-08-28/2.html

PISA PROgRAMME FOR INTERNATIONAL STUDENT ASSESSMENT. (2002). Reading for Change. Performance and Engagement across Countries. Results from PISA 2000. Nueva York: OECD.

Polinsky, M., y Kagan, O. (2007). «Heritage Languages: In the Wild' and in the Classroom.», Language and Linguistics Compass, 1 (5), pp. 368-395.

Propuestas de Alicante (2006). Recuperado de: http://segundaslenguaseinmigracion.com/inicio/propuestasalicante.pdf

Pugliese, R. y Malavolta, S. (2017). «La pluralità linguistica in classe come risorsa per le attività riflessive». En M. Vedovelli (ed.), L'italiano dei nuovi italiani (pp. 269289). Roma, Italy: Aracne.

Rasinski, T., Samuels, S. J., Hiebert, E., Petscher, Y. y Feller, K. (2011). «The Relationship between a Silent Reading Fluency Instructional Protocol on Students' Reading Comprehension and Achievement in an Urban School Setting», Reading Psychology, 32 (1), pp. 75-97.

REDER, S. y BYNNER, J. (2009). «The Need for Longitudinal Studies in Adult Literacy and Numeracy Education». En S. Reder y J. Bynner. (eds.), Tracking Adult Literacy and Numeracy Skills. Findings from Longitudinal Research (pp. 1-26). London: Routledge.

Rocca, L., CARlsen, C. H., y Deygers, B. (2019). The 2018 Council of Europe \& ALTE Survey on Language and Knowledge of Society Policies for Migrants. Consejo de Europa.

Rodrigo, V., Greenberg, D., Burke, V., Hall, R., Berry, A, Brinck, T., JosePh, H. y OBY, M. (2007). «Implementing an Extensive Reading Program and Library for Adult Literacy Learners», Reading in a Foreign Language, 19, pp. 106-119.

Ruiz, R. (1984). «Orientations in Language Planning», The Journal for the National Association for Bilingual Education, 8(2), pp. 15-34.

Schwartz, M., Share, D. L., Leikin, M., y Kozminsky, E. (2007). «On the Benefits of Bi-literacy: Just a Head Start in Reading or Specific Orthographic Insights?», Reading and Writing, 21, pp. 905-927.

Segalowitz, N., Poulsen, C., y Komoda, M. (1991). «Lower Level Components of Reading Skill in Higher Level Bilinguals: Implications for Reading Instruction». AILA Review, 8 (1), 15-30.

Shameem, N., McDermott, K., MArtin-Blaker, J, y CARryer, J. (2002). A Report on the Literacy Gains of Low-level and Pre-literate Adult Learners in Literacy Classes. Executive Summary. Nueva Zelanda: National Association of ESOL Home Tutor Schemes Inc, School of English \& Applied Linguistics UNITEC Institute of Technology Centre for Refugee Education, Auckland U. of Technology.

SHIN, S. J. (2013). Bilingualism in Schools and Society: Language, Identity and Policy. New York, NY: Routledge.

Simpson, J., y Whiteside, A. (eds.) (2015). Adult Language Education and Migration. Challenging Agendas in Policy and Practice. London, UK: Routledge. 
SosinsKi, M. (2020). «Reading from a Psycholinguistic Perspective». En J. Kreeft Peyton y M. Young-Scholten (eds.), Teaching Adult Immigrants with Limited Formal Education (pp. 30-51). Bristol: Multilingual Matters.

SMYSER, H. (2016). The Goldilocks of Variability and Complexity: The Acquisition of Mental Orthographic Representations in Emergent Refugee Readers. Tucson, AZ: University of Arizona.

Strucker, J. y DAvidson, R. (2003). The Adult Reading Components Study. Cambridge, MA: National Center for the Study of Adult Learning and Literacy.

TAMMELIN-LAine, T. (2014). «Aletaan alusta. Luku- ja kirjoitustaidottomat aikuiset uutta kieltä oppimassa. [Let's start from the beginning. Non-literate adults learning a new language.]», Jyväskylä Studies in Humanities, 240. Jyväskylä: Universidad de Jyväskylä.

TARONE, E. y Bigelow, M. (2005). «Impact of Literacy on Oral Language Processing: Implications for SLA Research». Annual Review of Applied Linguistics, 25(1), pp. 7797.

TAYLOR, M. (1992). The Language Experience Approach and Adult Learners. Recuperado de http://www.cal.org/caela/esl_resources/digests/LEA.html

Thomas, W. P., y Collier, V. (1997). School Effectiveness for Language Minority Students. Washington, DC: National Clearinghouse for Bilingual Education.

Toolan, M. (2009). Narrative Progression in the Short Story. A Corpus Stylistic Approach. Amsterdam: Benjamins.

VAinikKa, A. y Young-Scholten, M. (2011). The Acquisition of German: Introducing Organic Grammar. Berlin: de Gruyter.

VAn DE CraAts, I. y Young-Scholten, M. (2015). «Developing Technology Enhanced Literacy Learning for LESLLA Learners». En M. Santos y A. Whiteside. (eds.), Low Educated Second Language and Literacy Acquisition: Proceedings of the Nineth Symposium (pp. 129-150). San Francisco: Lulu Publishing Services.

VILAR, M. (Coord.) (2008). Oralpha. Método de alfabetización y comunicación oral en castellano y catalán significativo. Barcelona: Comissió de Formació ACOF.

VILlalBA, F. (2019). «Inmigración y enseñanza a adultos». En J. Muñoz-Basols, E. Gironzetti, M. Lacorte (eds.), The Routledge Handbook of Spanish Language Teaching (pp. 463-476). Peter Lang.

VillalBA, F. y HERnÁNDEZ, M.T. (2000). «¿Se puede aprender una segunda lengua sin saber leer? Alfabetización y aprendizaje de una L2». Carabela, 48, pp. 85-110.

VillalBA, F. (2010). «La enseñanza de segundas lenguas a jóvenes y adultos inmigrantes». En Multilingüisme i pràctica educativa: VI Simposi: llengua, educación $i$ immigració, ed. Institut de Ciències de l'Educació Josep Pallach (pp. 69-95). Girona: Universitat de Girona.

VV. AA. (2000). La comprensión lectora en el aula de E/LE. Carabela, 48. Madrid: SGEL.

WagneR, D., y VenezKy, R. L. (1999). «Adult Literacy: The Next Generation», Educational Researcher, 28, pp. 21-29. 
WiLliamson, E. (2013). «Our Lives Press. Inspiring through the Experience of Others». National Association of English and Community Language Teaching to Adults News, $101,5$.

YADEN, D. B., MADRIGAL, P., y TAM A. (2003). «Access to Books and beyond: Creating and Learning from a Book Lending Program for Latino Families in the Inner City». En G. G. Garcia. (ed.), English Learners. Reaching the Highest Level of Literacy (pp. 357386). Newark, DE: IRA.

Young-Scholten, M. y NAEB, R. (2010). «Non-Literate L2 Adults' Small Steps in Mastering the Constellation of Skills Required for Reading». In T. Wall y Leong, M. (ed.) Low Educated Adult Second Language and Literacy (pp. 80-91). Calgary: Bow Valley College.

Young-Scholten, M., NAeB, R y Sosinski, M. (2020). «Antiguas y nuevas ideas para el apoyo educativo de los migrantes adultos vulnerables». En N. Gómez López y J. M. Fernández Campoy. (eds.), Educación en grupos vulnerables. Madrid: Octaedro.

Young-Scholten, M. y Strom, N. (2006). «First-time L2 Readers: Is There a Critical Period?». En J. Kurvers, I. van de Craats y M. Young-Scholten. (eds.), Low Educated Adult Second Language and Literacy Acquisition. Proceedings of the Inaugural Conference (pp. 45-68). Utrecht: LOT.

Young-Scholten, M., Sosinski, M. y Rubio, A. M. (2015). «Undergraduates' Involvement in Producing Short Fiction Books for Immigrant Adult Beginners in England and Spain». Language Issues: The ESOL Journal, 26(1), pp. 55-60.

Young-SChOlTEN, M. (2017). LESLLA-Based Survey of Teachers' Pleasure/Extensive Reading Practices Results. Manuscrito no publicado. 